\title{
Maximum Node Degree Mobility Metric for Wireless Ad hoc Networks
}

\author{
Arta Doci \\ Colorado School of Mines \\ adoci@mines.edu \\ William Springer \\ Colorado State University \\ wmspring@CS.ColoState.EDU \\ Fatos Xhafa \\ Technical University of Catalonia \\ fatos@1si.upc.edu
}

\begin{abstract}
Mobility has a significant impact on the ad hoc network protocol performance. Mainly, the protocol performance has been evaluated in simulations and using synthetic mobility models, which have two main drawbacks: (a) they assume that wireless devices start and remain in the simulation for a user defined simulation time; and, (b) they are unrealistic. Real mobility models that are derived from real user traces challenge the assumption that wireless devices start and remain in the simulation for the entire user defined simulation time, but they rather show that wireless nodes posses dynamic membership (nodes join and leave the simulation dynamically based on some random variable). In this paper, we evaluate the Maximum Node Degree mobility metric for real mobility models, which has got little attention due to the assumption of static connectivity graph on the number of nodes. The contributions of this paper are two-fold. First, we introduce the algorithm that computes the Maximum Node Degree mobility metric, which in turn provides the upper bound on the number of neighbors for a given node. Second, we show that the upper bound can be used to improve the algorithm complexity by introducing a new algorithm metric, namely Efficiency. Its usefulness is shown through a case study for evaluating the gains in the algorithm complexity of incentive protocols.
\end{abstract}

\section{Introduction}

Many studies have demonstrated that mobility has a significant impact on the performance of ad hoc network protocols. Specifically, the authors of [5] provide a framework, which is helpful in understanding how and what mobility characteristics affect the protocol performance. For example, the mobility metrics that effect the performance are average shortest path hop count $\left(A_{s p} H o p s\right)$ [10], average link duration [2] and average path durations [12]. In addition, other path and link metrics have been proposed by the authors of $[14,8,7]$ and have been shown to effect performance. All these mobility metrics are derived from synthetic mobility models, which face two main issues.

First, the connectivity graph, where the mobile nodes are the vertices and the communication links are the edges, have been assumed to be static on the number of mobile nodes. The number of vertices $|V|$ represent the order and the number of edges $|E|$ represent the size of the connectivity graph. In general, the running time of the algorithms are measured in terms of the order and size of the graph, which is relatively easy to estimate when the graph is static, but becomes cumbersome task when the graph is dynamic.

Second, the current implementations of the synthetic mobility models place the wireless nodes to start the simulation at time 0 and remain in the simulation until the allotted simulation time is over. On the other hand, real mobility models [15], which are extracted from real user traces, show that wireless nodes posses dynamic membership, that is, they join and leave the simulation based on an exponentially distributed random variable. Under dynamic membership of the nodes, the connectivity graph is dynamic on the number of vertices and edges. When adding the new dimension of the dynamic membership to simulation mobility models, then the mobility metrics need to be re evaluated under the new dimension, thus is the focus of this paper.

Maximum Node Degree mobility metric, which represents the maximum number of neighbors for each node (in graph theory terms it is the number of edges incident to it), can be used to account for the dynamic membership of the wireless nodes. The contributions of this paper are:

1. Design an optimal algorithm to compute Maximum Node Degree mobility metric.

2. Propose a new metric, namely Efficiency, which uses the Maximum Node Degree metric to introduce im- 
provements of the running time of the algorithms. We illustrate the efficiency by using as a case study the incentive ad hoc protocols.

\section{RealMobGen: A Real Mobility Model}

The main characteristics of a mobility model are speed, pause distribution, and direction of movement. For example, the most used synthetic mobility model is Random Walk Model (RWM) [3], which works as follows: Each node is assigned a randomly distributed initial location $(x 0 ; y 0)$; Then, each node randomly picks up a destination independent of their initial positions and moves toward it with speed chosen uniformly on the interval $(v 0 ; v 1)$; Nodes pause upon reaching each destination; Repeat the process until the allotted simulation time. There are several issues with RWM, which are addressed in RealMobGen.

RealMobGen is a hybrid model that is based on Dartmouth's model of mobile network traces [9] and USC's WWP [6] survey. The model mimics the environments where ad hoc networks will likely be deployed closer, since it borrows its characteristics from models derived from real user traces. Another feature of RealMobGen, that is not existent in any other current mobility model, is the classification of nodes as stationary ( $46 \%$ of the nodes) or mobile ( $54 \%$ of the nodes). The ratio of stationary vs. mobile nodes was borrowed from the Dartmouth model.

The stationary nodes select a location based on a transition matrix that defines the probabilities for moving from one point to another. Once a location is selected, a node is turned on for a time drawn from the exponential distribution of start time for the stationary nodes. Stationary node stays at the selected location until the allotted stationary end time. The mobile nodes, also, select a start location based on the transition matrix. The mobile node enters the simulation at a time drawn from the mobile node start time. The node pauses at the selected location until the allotted pause time from mobile pause time exponential distribution. After the pause time is up the mobile node selects the next location based on the transition matrix and moves there not in straight line but following data that supports movements along popular roads and turns. The mobile node repeats the pattern 'pause-select next location - move there' until allotted mobile simulation end time.

RealMobGen shows that wireless nodes tend to cluster around popular locations, i.e., cafeteria, gym, classes, and library. We believe that RealMobGen is the first mobility model (we are not aware of any other models) that implements the dynamic characteristic of wireless devices in NS 2, devices join and leave the network at different times. RealMobGen addresses the drawbacks present in the RWM by implementing the following new features:

Feature 1: Wireless Nodes are clustered around popular hotspots. For example, Figure ?? shows a snapshot of RealMobGen with 40 nodes, which are clustered around 14 hotspots.

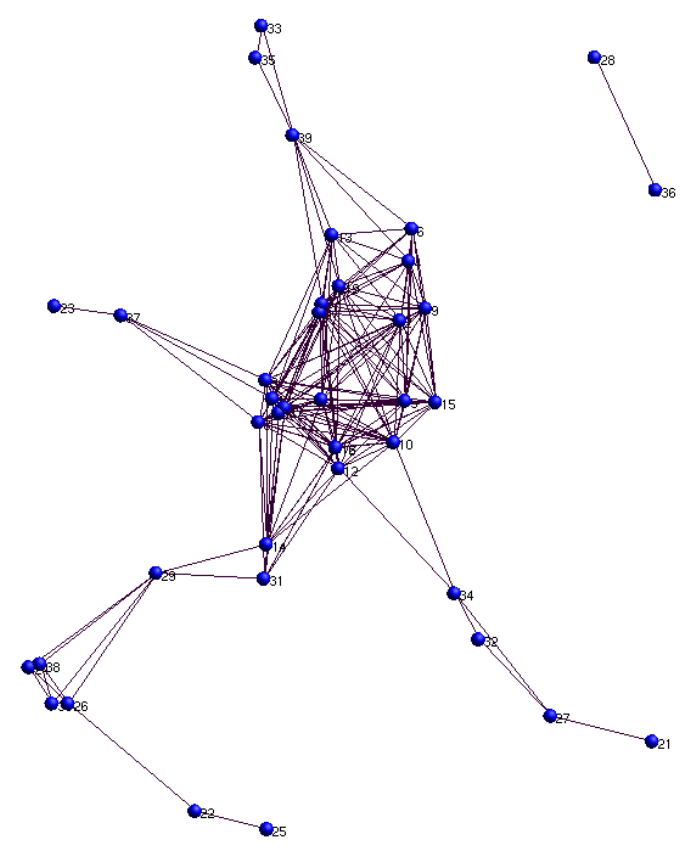

Figure 1. 40 wireless nodes clustered around 14 Hotspots.

Feature 2: Wireless Nodes posses dynamic membership. For example, Figure 2 shows the dynamic membership of 60 nodes.

Feature 3: Nodes are classified on two flavors, namely stationary and mobile (stationary $(46 \%)$ of the nodes or mobile ( $54 \%$ of the nodes). The ratio of stationary vs. mobile nodes was borrowed from the Dartmouth model.

Feature 4: Moving from one point to another is done via waypoints, instead of a straight line.

\section{Maximum Node Degree}

In real mobility models the connectivity graph $G=$ $(V, E)$ is a dynamic graph on vertices that join and leave and edges and edges that appear and disappear due to mobility. In graph theory terms, when taking into account the dynamic membership of the nodes, there is an edge between any two nodes $(i, j)$, if the following two conditions are met:

1. $D_{i, j} \leq R$, where $\mathrm{D}$ is the euclidian distance between nodes $(i, j)$ and $\mathrm{R}$ is the transmission range of the wireless nodes. 


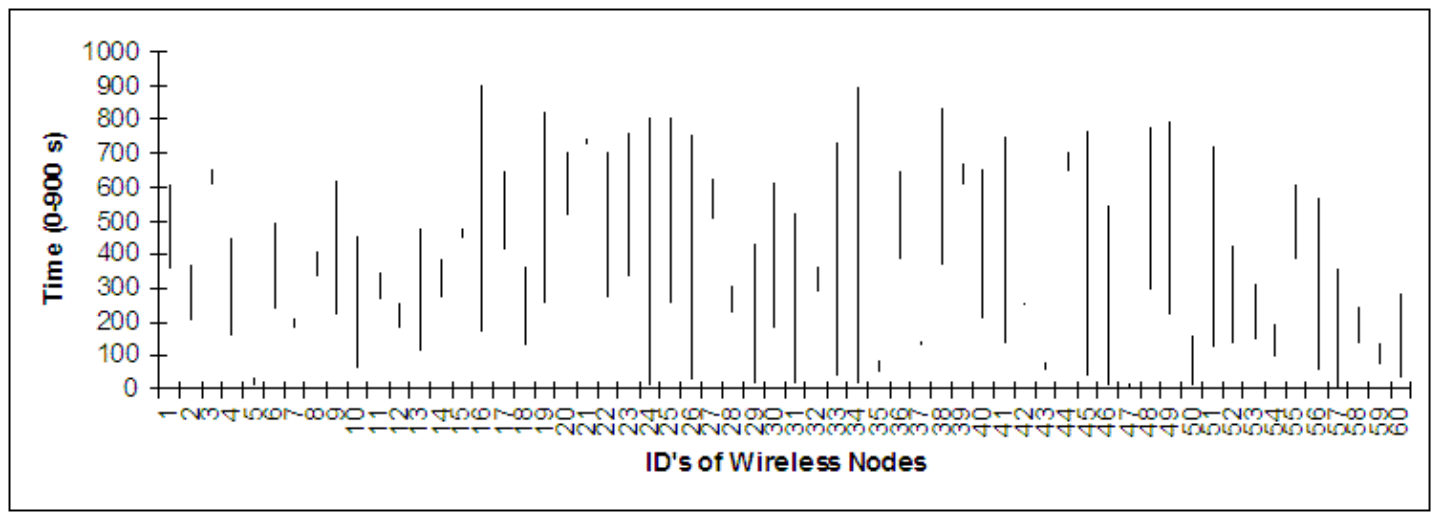

Figure 2. Dynamic Membership of 60 Nodes.

2. $I_{i} \cap I_{j} \neq \emptyset$, where I represents the active intervals of the nodes.

Thus, when interested on the mobility metrics, i.e., path and link duration, we are basically posing the shortest path problem in the case of dynamic topology and links. Basically, this can be represented as a graph with edges that are added and removed, as the vertices turn 'on' and 'off'. While the method of calculating the full graph is polynomial in the size of the input, it is quite inefficient as each movement of a node can potentially cause $n-1$ edge insertions and the same number of deletions. We can obtain better bounds by restricting the algorithm to only processing the nodes, which are currently relevant.

In order to consider only the nodes that are relevant, we simplify the problem by first processing the dynamic membership information. For example, consider two nodes $i$ and $j$. Since all communication is instantaneous, any communication between the nodes must occur while both are turned on; if communication is indirect, then every node that participates in routing the messages must also be turned on during that period of time. While, we should note that, this can be $O(n)$ nodes in the worst case, in most cases this allows us to consider only a fraction of the full graph.

\subsection{Dynamic Membership Graph is an In- terval Graph}

Given a set of $n$ intervals (which represent the number of wireless nodes) $I=I_{1}, I_{2}, \ldots, I_{n}$, the corresponding interval graph $G=(V, E)$ has the set of vertexes $V=$ $v_{1}, v_{2}, \ldots, v_{n}$ and there is an edge $E$ linking nodes $\left(v_{i}, v_{j}\right)$ if and only if $I_{i} \cap I_{j} \neq \emptyset$ (see Figure 3). We will use the interval graphs to calculate the MaximumNodeDegree.

In order to define the upper bounds of the number of nodes that are neighbors, we formulate the problem as a coloring problem, where the goal of the algorithm is to color

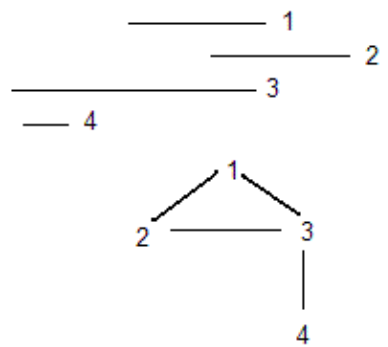

Figure 3. A set of four intervals and the corresponding interval graph.

each interval with the minimal number of colors in such a way that no two overlapping intervals are colored with the same color. The minimal number of colors represents the upper bound of the Maximum Node Degree ${ }^{1}$. In Figure 4, we graph the dynamic membership information, as an interval graph, which is parsed from the output of RealMobGen with 40 wireless nodes.

\subsection{Algorithm for Maximum Node Degree}

In this section we present the algorithm for the Maximum Node Degree. The algorithm reads each interval and sorts them by the beginning interval times (see Figure 4).

The MaximumNodeDegree is derived by the GreedlyMaximumNodeDegree algorithm (see Algorithm 1). The input to the algorithm is the dynamic membership of the nodes, which is a series of intervals that are determined by their beginning and ending time of active nodes. Our goal is to find all the overlapping active times intervals. The number of overlapping intervals gives us the

\footnotetext{
${ }^{1}$ The algorithm in this section is similar to independent work in the context of channel routing problem [13].
} 


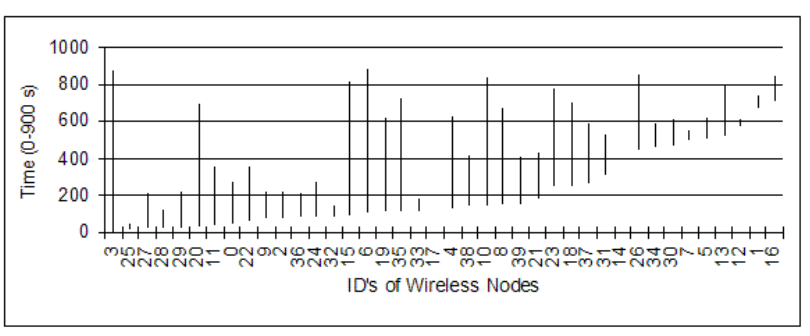

Figure 4. Dynamic Membership Graph on $\mathbf{4 0}$ nodes: Sorted by beginning interval times.

upper bound of the number of neighbors for each node. We set the problem as a coloring problem. The goal is to color each interval with the minimal number of colors in such a way that no two overlapping intervals are colored with the same color. First, the algorithm reads all the intervals and sorts them by their their beginning times. After it assigns to the first interval the Color 1, the algorithm looks at the second interval, in order to figure out whether it overlaps with any other intervals. If it overlaps, then it calculates what colors have already been used and assigns the next available color to the interval. On the other hand, if the answer is "no", then the algorithm assigns the color of the overlapping interval.

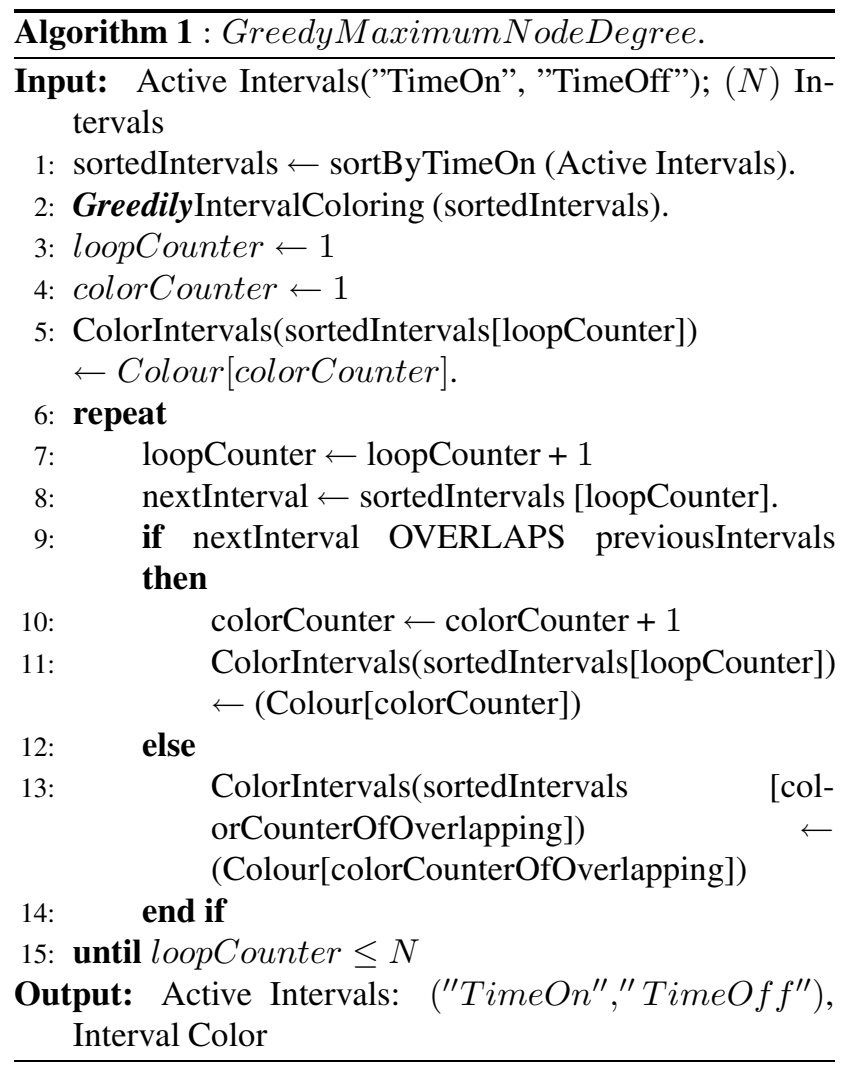

We illustrate the algorithm by running it in the same example of the 40 nodes used in the previous sections. For example, in Table 1 we show the partial output of the algorithm. The table illustrates that the first seven intervals overlap, thus the color of the intervals is incremental. On the other hand, the eighth interval defined by $(39.14919133,269.6973103)$ does not overlap with the second one $(9.112384965,35.76696803)$, thus gets assigned the same color (Color 2).

The algorithm Greedy MaximumNodeDegree falls in the greedy class of the algorithms, since it takes the best immediate, or local, solution while finding a global one. The global solution represents the upper bound of the node neighbors degree, while the average of the local optimum(s) and the global one provides us with the average node neighbors degree.

It can be shown that the algorithm is optimal (following a well known result from greedy scheduling algorithms; we omit the details).

\section{Efficiency using Maximum Node Degree}

In order to demonstrate how to use the Maximum Node Degree mobility metric to improve algorithm efficiency we illustrate it by a case study that is the incentive protocols in ad hoc networks.

\subsection{Incentive Ad Hoc Protocols}

Ad hoc networks are self-organizing and multi-hop networks with no central authority. Thus, every aspect of the configuration and operation of an ad-hoc network is distributed. Another characteristic is that nodes are power and energy constrained. Thus, each node running a distributed protocol must make its own decisions (possibly relying on information from other nodes). Those decisions maybe constrained by the rules or algorithms of a protocol, but ultimately each node would have some leeway in setting parameters or changing the mode of operation. These nodes are autonomous agents, making decisions about transmit power, packet forwarding, back off time, and so on.

In the presence of the selfish nodes, the goal of each wireless device is to maximize its welfare: WELFARE = Profit-Costs, where profit is the payments received for forwarding traffic and the costs are the incurred energy loss of the node by transmitting packets for other nodes and sending its own traffic; and the payments to others that forwarded its own traffic. On the other hand the goal of the incentive protocol designer is to provide incentives to the wireless nodes to relay traffic, such that nodes will have no incentives to deviate from the protocol, since doing so will not bring them a higher welfare. 
Table 1. The partial output of the Algorithm GreedyMaximumNodeDegree.

\begin{tabular}{ccc}
\hline Time node turns “ON" & Time node turns “OFF" & Assigned Interval Color \\
\hline 1.28239834 & 874.2507994 & 1 \\
9.112384965 & 35.76696803 & 2 \\
12.33443828 & 206.6781021 & 3 \\
13.96709987 & 116.2303641 & 4 \\
15.42727166 & 212.4065595 & 5 \\
22.61315476 & 686.9883725 & 6 \\
28.87034892 & 349.2144535 & 7 \\
39.14919133 & 269.6973103 & 2 \\
\hline
\end{tabular}

In the presence of selfish nodes most of the proposed protocols are based on the well known Vickrey-Clarke-Groves (VCG) mechanism. Below we describe one such protocol based on VCG. Vickery auction is most familiar because it is the foundation of eBay's auction design. In the Vickrey auction the high bidder wins, but pays the second-highest bid. This is why the Vickrey auction is called a second-price auction: the price is not the highest bid, but the secondhighest bid. Desirable properties of auction protocols are given in [11], which include Strategy-Proofness, Pareto Efficiency, Individual Rationality, and Budget-Balance.

In $[1,4,16]$ the authors propose incentive protocols that ensure that the participating wireless nodes will have no reason to deviate from the protocol, since doing so will not bring them a higher welfare. For example, in [1] the authors propose the Ad-Hoc VCG protocol, which is a reactive routing protocol that achieves the design objectives of truthfulness. Reactive protocols seek to set up routes on-demand, thus topology information is only transmitted by nodes ondemand. In this protocol vertexes represent the nodes and weighted directed edges represent the payments an emitting node has to receive. Nodes are awarded payments for forwarding a message, thus cover the cost for forwarding a unit-size packet: Payment $=c_{i} * P^{\text {emit }}$, where $c_{i}$ is the cost-of energy of $\$ / W a t t$ and $P^{e m i t}$ is emission signal strength in watt. The protocol can be thought as it is run on two phases. Firstly, route discovery, where nodes communicate to destination $P^{e m i t}$ and $c_{i}$. Then, the destination computes Lowest Cost Path (LCP). Secondly, data transmission, packets are forwarded along the shortest path route and payments are made to the intermediate nodes. Payment = DeclaredCost $+\left(L C P_{\text {withthenode }}-L C P_{\text {withoutthenode }}\right)$.

The algorithm complexity for the proposed protocols [1, 16] is $O\left(N^{3}\right)$, for each time snap of the network during simulation time. In [4] the authors try to reduce the complexity to $O\left(M^{2} * d\right)$, where $\mathrm{d}$ is the diameter and $\mathrm{M}$ is some upper bound for the node degree. However, the value of $\mathrm{M}$ was not computed. We computed $\mathrm{M}$ by using the GreedyMaximumNodeDegree Algorithm. Next, we
Table 2. RealMobGen Simulation Parameters.

\begin{tabular}{ll}
\hline Parameter & Value \\
\hline Simulation Duration & $900 \mathrm{~s}$ \\
Simulation Area & $900 \times 1200 \mathrm{~m}$ \\
Number of Hotspots & 14 \\
Number of Nodes (nn) & $n n \in(40,60,100)$ \\
\hline
\end{tabular}

present the efficiency introduced by taking into account the value of $\mathrm{M}$, which is the maximum node degree.

\subsection{Efficiency}

We propose the metric Efficiency, as a metric to evaluate the gains in the algorithm complexity of incentive protocols. In this section, we run the GreedlyDynamicMembership with several scenarios. The scenarios are generated using RealMobGen mobility model. The inputs to the RealMobGen are the simulation duration set to 900 seconds; simulation area of $900 \times 1200$ meters; number of hotspots set to 14 , while the number of nodes was varied 40,60, 100 (the parameters are summarized in Table 2).

For each set of nodes, we run the simulation 10 times and present the results in Table 3. We define a new metric, namely Efficiency, that calculates the improvement of real mobility metric algorithms over the synthetic ones, i.e., in terms of relevant comparisons (see Equation (1)). For example, from the table we have the Ef ficiency for 40,60, 100 number of nodes to be respectively $54.75,54.33,52.30$, which demonstrates that we need to compare only half of the nodes, instead of the full graph when computing the mobility metrics $\left(N^{2}\right)$.

$$
\text { Efficiency }=\left(\frac{\frac{\sum_{T} \text { MaxNodeD } D_{\text {egree }}}{T}}{N}\right) * 100 .
$$


Table 3. Number of necessary comparisons on 40, 60, 100 nodes.

\begin{tabular}{ccc}
\hline MaxNode $D_{\text {egree }}$ on 40 Nodes & MaxNode $D_{\text {egree }}$ on 60 Nodes & MaxNode $D_{\text {egree }}$ on 100 Nodes \\
\hline 24 & 33 & 50 \\
21 & 27 & 55 \\
16 & 28 & 49 \\
25 & 35 & 55 \\
21 & 32 & 53 \\
25 & 34 & 55 \\
20 & 37 & 48 \\
23 & 32 & 51 \\
21 & 29 & 54 \\
23 & 39 & 53 \\
\hline \hline Efficiency $=54.75 \%$ & Efficiency $=54.33 \%$ & Efficiency $=52.30 \%$ \\
\hline
\end{tabular}

\section{Conclusions}

In this paper we demonstrated that when introducing dynamic topology the current mobility metrics need to be re evaluated. We introduced Maximum Node Degree as a mobility metrics, which can be used to improve algorithm efficiency. In the future, we plan to re evaluate all the current mobility metrics and provide the lower bounds for node degrees. Furthermore, we plan to propose a new protocol that involves mobility in the design choice.

\section{Acknowledgments}

Research partially supported by ASCE TIN2005-09198C02-02 and FP6-2004-IST-FETPI (AEOLUS) Research Projects.

\section{References}

[1] L. Anderegg and S. Eidenbenz. Ad hoc-vcg: a truthful and cost-efficient routing protocol for mobile ad hoc networks with selfish agents. In MobiCom, Septmber 2003.

[2] J. Boleng, W. Navidi, and T. Camp. Metrics to enable adaptive protocols for mobile ad hoc networks. In International Conference onWireless Networks, pages 293-298, 2002.

[3] J. Broch, D. Maltz, D. Johnson, Y. Hu, and J. Jetcheva. Multihop wireless ad hoc network routing protocols. In MobiCom 1998: Proceedings of the Fourth Annual ACM International Conference on Mobile Computing and Networking, page 8597, 1998.

[4] S. Eidenbenz, G. Resta, and P. Santi. Commit: A sendercentric truthful and energy-efficient routing protocol for ad hoc networks with selfish nodes. In IPDPS, April 2005.

[5] F.Bai, N. Sadagopan, and A. Helmy. Important: a framework to systematically analyze the impact of mobility on performance of routing protocols for adhoc networks. In INFOCOM, pages 825-835, 2003.

[6] W. J. Hsu, K. Merchant, H. W. Shu, C. H. Hsu, and A. Helmy. Weighted waypoint mobility model and its impact on ad hoc networks. SIGMOBILE Mobile Computer Communications Review, 9(1):59-63, 2005.

[7] S. Jiang. An enhanced prediction-based link availability estimation for manets. IEEE Transactions on Communications, 52(2):183-186, 2004.

[8] H. M. Jones, S. Xu, and K. Blackmore. Link ratio for ad hoc networks in a rayleigh fading channel. In WITSP, pages 252-255, 2004.

[9] M. Kim, D. Kotz, and S. Kim. Extracting a mobility model from real user traces. In INFORCOM: Proceedings of the 25th Annual Joint Conference of the IEEE Computer and Communications Societies, pages 1-13, 2006.

[10] S. Kurkowski, W. Navidi, and T. Camp. Constructing manet simulation scenarios that meet standards. In MASS, page To Appear, 2007.

[11] A. Mas-Colell, M. D. Whinston, and J. R. Green. Microeconomic theory. Oxford University Press, 1995.

[12] N. Sadagopan, F. Bai, B. Krishnamachari, and A. Helmy. Paths: analysis of path duration statistics and their impact on reactive manet routing protocols. In MobiHoc, pages 245256, 2003.

[13] M. Sarrafzadeh and C. K. Wong. An Introduction to VLSI Physical Design. McGraw-Hill Higher Education, 1996.

[14] W. Su, S. Lee, and M. Gerla. Mobility prediction and routing in ad hoc wireless networks. International Journal of Network Management, 11(2):3-30, 2001.

[15] C. Walsh, A.Doci, and T.Camp. A call to arms: It's time for real mobility models. In MC2R Special Issue based on Student Posters at MobiCom 2007.

[16] S. Zhong, L. E. Li, Y. G. Liu, and Y. R. Yang. On designing incentive-compatible routing and forwarding protocols in wireless ad-hoc networks: an integrated approach using game theoretical and cryptographic techniques. In MobiCom, pages 117-131, 2005. 